\title{
THE CRITICAL NUMBERS FOR UNSYMMETRICAL APPROXIMATION
}

RAPHAEL M. ROBINSON

1. Statement of the main theorem. If $\xi$ is an irrational number, then the modulus of approximability from the right, $M^{+}(\xi)$, is defined as the least upper bound of the values of $\mu$ for which the inequality

$$
0<\frac{A}{B}-\xi<\frac{1}{\mu B^{2}}
$$

has infinitely many solutions. In a similar way, $M^{-}(\xi)$ is defined measuring the approximability of $\xi$ from the left.

The number $\xi$ is called critical if there is no other irrational number $\xi^{\prime}$ for which

$$
M^{+}\left(\xi^{\prime}\right)<M^{+}(\xi), \quad M^{-}\left(\xi^{\prime}\right)<M^{-}(\xi) .
$$

That is, roughly speaking, $\xi$ is called critical if there is no other number which is harder to approximate both from the right and from the left. The purpose of this paper is to give a necessary and sufficient condition that $\xi$ be critical.

A few definitions are necessary before stating the main theorem. A sequence of non-negative integers $r_{1}, r_{2}, r_{3}, r_{4}, \ldots$ will be called derivable if

$$
\lim \inf r_{n}+1=\lim \sup r_{n}<\infty,
$$

that is, if ultimately just two different numbers occur in the sequence, and these are consecutive integers $k$ and $k+1$. In this case, the sequence has the form

$$
r_{1}, r_{2}, \cdots, r_{v}, k,(k+1)^{s_{1}}, k,(k+1)^{s_{2}}, k,(k+1)^{s_{3}}, k, \cdots,
$$

the exponents denoting repetition of the term $k+1$. Here the $s_{n}$ are non-negative integers; if there are consecutive $k$ 's in the sequence, then we must take some of the $s_{n}$ equal to zero.

We shall call $s_{1}, s_{2}, s_{3}, \cdots$ the derived sequence. Together with $k$, this derived sequence determines the end of the primitive sequence:

$$
k,(k+1)^{s_{1}}, k,(k+1)^{s_{2}}, k,(k+1)^{s_{3}}, k, \cdots .
$$
1947.

Presented to the Society, October 25, 1947; received by the editors, August 25, 
It will be convenient in the proof to know that the preceding term $r_{v}$ is also either $k$ or $k+1$. For definiteness, we may suppose that $v$ is chosen as small as possible so that this is true.

If the derived sequence is derivable, its derivative will be called the second derivative of the primitive sequence, and likewise for higher derivatives. The primitive sequence itself will be called the zeroth derivative.

MAIN TheOREM. The number $\xi$ is critical if and only if its expansion as a continued fraction has the form

$$
\xi=\left[q_{0}, q_{1}, \cdots, q_{u-1}, 1, r_{1}, 1, r_{2}, 1, r_{3}, 1, \cdots\right],
$$

where the sequence $r_{1}, r_{2}, r_{3}, \cdots$ either has infinitely many derivatives, or its highest derivative is a sequence

$$
t_{1}, t_{2}, t_{3}, \cdots
$$

such that $t_{n} \rightarrow k$, where $k$ is a positive integer, or $t_{n} \rightarrow \infty$.

2. Discussion of the theorem. By $M(\xi)$ is meant the least upper bound of numbers $\mu$ such that the inequality

$$
\left|\frac{A}{B}-\xi\right|<\frac{1}{\mu B^{2}}
$$

has infinitely many solutions. Thus

$$
M(\xi)=\max \left\{M^{+}(\xi), M^{-}(\xi)\right\} .
$$

The condition that $\xi$ be critical is strikingly similar to the known condition that $M(\xi) \leqq 3$. We have indeed $M(\xi) \leqq 3$ if and only if the continued fraction expansion of $\xi$ has the form

$$
\xi=\left[q_{0}, q_{1}, \cdots, q_{u-1}, r_{1}, r_{1}, r_{2}, r_{2}, r_{3}, r_{3}, \cdots\right],
$$

where each $r_{n}$ is either 1 or 2 , and the sequence $r_{1}, r_{2}, r_{3}, \cdots$ satisfies the same conditions as in the theorem. ${ }^{1}$ Despite the similarity of the results, no connection is apparent between the problems, and the methods

1 This result does not appear in the literature in exactly this form. However, it is closely related to results obtained by A. Markoff, Sur les formes quadratiques indéfinies, Math. Ann. vol. 15 (1879) pp. 381-406 and vol. 17 (1880) pp. 379-399. While the problem he considered was different, the methods and results can be carried over to the problem concerning $M(\xi) \leqq 3$. (Conversely, the main theorem of this paper could be carried over into a theorem concerning the numerically smallest positive and negative values of an indefinite quadratic form.) See also P. J. Heawood, The classification of rational approximations, Proc. London Math. Soc. (2) vol. 20 (1921) pp. 233250. 
used to establish the two results are different.

In a previous paper, ${ }^{2}$ which will be referred to as I, we showed how to determine the values of $M^{+}(\xi)$ and $M^{-}(\xi)$ from the continued fraction for $\xi$. Indeed, if

$$
\xi=\left[q_{0}, q_{1}, q_{2}, q_{3}, \cdots\right]
$$

and we set

$$
\lambda_{n}=\left[q_{n+1}, q_{n+2}, q_{n+3}, \cdots\right]+\left[0, q_{n}, q_{n-1}, \cdots, q_{1}\right],
$$

then

$$
M^{+}(\xi)=\lim \sup \lambda_{2 n+1}, \quad M^{-}(\xi)=\lim \sup \lambda_{2 n} .
$$

The proof of the theorem will depend entirely on a study of the $\lambda_{n}$.

A special notation was introduced for a sum of two continued fractions, such as $\lambda_{n}$. We write

$$
\lambda_{n}=\left[q_{1}, q_{2}, \cdots, q_{n},\left[q_{n+1}\right], q_{n+2}, q_{n+3}, \cdots\right] .
$$

This expression will be called a double continued fraction, and will be said to be centered at $q_{n+1}$, or $q_{n+1}$ will be called the marked term.

A knowledge of $\mathrm{I}, \S \S 1-5$, is required for the present paper. However, $\$ \S 3-5$ below are independent of $\mathrm{I}$.

3. The average of two continued fractions. Suppose that the two real numbers $\rho$ and $\sigma$ can be expanded in continued fractions

$$
\begin{aligned}
& \rho=\left[r_{0}, r_{1}, \cdots, r_{v-1}, \alpha\right], \\
& \sigma=\left[r_{0}, r_{1}, \cdots, r_{v-1}, \beta\right],
\end{aligned}
$$

which agree except for the last term, and in which all the terms are positive. In what form can we write the continued fraction for the average of $\rho$ and $\sigma$ ? We shall put

$$
(\rho+\sigma) / 2=\left[r_{0}, r_{1}, \cdots, r_{v-1}, \gamma\right],
$$

and investigate the value of $\gamma$. It is clear that $\gamma$ is between $\alpha$ and $\beta$. But we shall prove more, namely that $\gamma$ is between the harmonic and arithmetic means of $\alpha$ and $\beta$ :

$$
\frac{2 \alpha \beta}{\alpha+\beta} \leqq \gamma \leqq \frac{\alpha+\beta}{2} .
$$

We see that for $v=0, \gamma$ is equal to the arithmetic mean, and for $v=1$

${ }^{2}$ R. M. Robinson, Unsymmetrical approximation of irrational numbers, Bull. Amer. Math. Soc. vol. 53 (1947) pp. 351-361. References to the work of Segre and others will be found there. 
to the harmonic mean. Now suppose that the conclusion is known for some $v$, and prove it for $v+1$. We are given

$$
\begin{aligned}
& \rho=\left[r_{0}, \cdots, r_{v-1}, r_{v}, \alpha\right]=\left[r_{0}, \cdots, r_{v-1}, r_{v}+\alpha^{-1}\right], \\
& \sigma=\left[r_{0}, \cdots, r_{v-1}, r_{v}, \beta\right]=\left[r_{0}, \cdots, r_{v-1}, r_{v}+\beta^{-1}\right],
\end{aligned}
$$

and are to express their average in the form

$$
(\rho+\sigma) / 2=\left[r_{0}, \cdots, r_{v-1}, r_{v}, \gamma\right]=\left[r_{0}, \cdots, r_{v-1}, r_{v}+\gamma^{-1}\right] .
$$

We have, by the hypothesis of the induction,

$$
\frac{2\left(r_{v}+\alpha^{-1}\right)\left(r_{v}+\beta^{-1}\right)}{\left(r_{v}+\alpha^{-1}\right)+\left(r_{v}+\beta^{-1}\right)} \leqq r_{v}+\gamma^{-1} \leqq \frac{\left(r_{v}+\alpha^{-1}\right)+\left(r_{v}+\beta^{-1}\right)}{2} .
$$

The right side of the inequality is equivalent to $\gamma \geqq 2 \alpha \beta /(\alpha+\beta)$, and the left side when simplified gives

$$
\gamma \leqq \frac{\alpha+\beta+r_{v} \cdot 2 \alpha \beta}{2+r_{v}(\alpha+\beta)} .
$$

Since the right side here is between the arithmetic and harmonic means of $\alpha$ and $\beta$, the desired conclusion follows.

REMARK. If we wish to "symmetrize" the double continued fraction

$$
\left[\alpha, r_{v-1}, \cdots, r_{1},\left[r_{0}\right], r_{1}, \cdots, r_{v-1}, \beta\right]
$$

by replacing $\alpha$ and $\beta$ by one and the same number $\gamma$, then the required value of $\gamma$ is between the arithmetic and harmonic means of $\alpha$ and $\beta$.

4. Comparison of two double continued fractions. There is no simple rule for deciding in general which of two double continued fractions is the larger. We shall however give a rule which applies in certain cases where the central parts of the continued fractions are symmetrical.

Theorem. Suppose that $r_{0}, r_{1}, \cdots, r_{v-1}$, and $s$ are positive integers, and that $\alpha, \beta, \gamma$, and $\delta$ are real numbers greater than 1 . Let $R$ stand for the sequence, including a marked term,

$$
r_{v-1}, \cdots, r_{2}, r_{1},\left[r_{0}\right], r_{1}, r_{2}, \cdots, r_{v-1} .
$$

Then if $v$ is even, we shall have

$$
[\gamma, 1, s, R, s-1,1, \delta]<[\alpha, 1, s, R, s, 1, \beta],
$$

and provided $\alpha<4 s+3$ and $\beta<4 s+3$, also

$$
[\gamma, 1, s, R, s+1,1, \delta]>[\alpha, 1, s, R, s, 1, \beta] .
$$


If $v$ is odd, the inequalities are reversed.

Proof. It will be sufficient to consider the case in which $v$ is even. To prove each inequality, we start with the left side, and apply the results of $\$ 3$ to symmetrize.

The proof of the first inequality proceeds as follows:

$$
\begin{aligned}
{[\gamma, 1, s, R, s-1,1, \delta] } & <[s+1 ; R, s] \\
& \leqq[s+1 / 2, R, s+1 / 2]<[\alpha, 1, s, R, s, 1, \beta] .
\end{aligned}
$$

Here we symmetrized using the arithmetic mean. For the second inequality we have

$$
\begin{aligned}
{[\gamma, 1, s, R, s+1,1, \delta] } & >[s+1 / 2, R, s+3 / 2] \\
& \geqq[4 s+3,1, s, R, s, 1,4 s+3] \\
& >[\alpha, 1, s, R, s, 1, \beta] .
\end{aligned}
$$

Here we symmetrized by introducing the harmonic mean; a simple calculation shows that the harmonic mean of $s+1 / 2$ and $s+3 / 2$ is $[s, 1,4 s+3]$.

We shall now prove a generalization of this theorem. The following lemma is needed.

LEMMA. If

$\left[\cdots, r_{n-1},\left[r_{n}\right], r_{n+1}, r_{n+2}, \cdots\right]<\left[\cdots, r_{n-1}^{\prime},\left[r_{n}^{\prime}\right], r_{n+1}^{\prime}, r_{n+2}^{\prime}, \cdots\right]$ and

$$
\left[r_{n+1}, r_{n+2}, \cdots\right]>\left[r_{n+1}^{\prime}, r_{n+2}^{\prime}, \cdots\right]
$$

then

$$
\left[\cdots, r_{n-1}, r_{n},\left[r_{n+1}\right], r_{n+2}, \cdots\right]>\left[\cdots, r_{n-1}^{\prime}, r_{n}^{\prime},\left[r_{n+1}^{\prime}\right], r_{n+2}^{\prime}, \cdots\right] \text {. }
$$

Proof. Let $\rho=\left[r_{n+1}, r_{n+2}, \cdots\right]$ and $\sigma=\left[r_{n}, r_{n-1}, \cdots\right]$, and similarly for $\rho^{\prime}$ and $\sigma^{\prime}$. By hypothesis, $\rho>\rho^{\prime}$; thus if $\sigma \leqq \sigma^{\prime}$, the conclusion

$$
\rho+1 / \sigma>\rho^{\prime}+1 / \sigma^{\prime}
$$

is clear. On the other hand, if $\sigma>\sigma^{\prime}$, we have

$$
\rho+1 / \rho+\sigma+1 / \sigma>\rho^{\prime}+1 / \rho^{\prime}+\sigma^{\prime}+1 / \sigma^{\prime} .
$$

Subtracting the inequality

$$
\sigma+1 / \rho<\sigma^{\prime}+1 / \rho^{\prime}
$$

which is true by hypothesis, we again have the desired conclusion.

REMARK. Thus if the order of two double continued fractions is 
what would be expected by looking only at the part to the right of the marked term, then when the marked term is shifted one place to the right in each continued fraction, the order is reversed.

Using this lemma, we may generalize the preceding theorem. A remark is necessary concerning a notation used in the extended theorem. If $b_{1}, \cdots, b_{s}$ is a sequence of numbers, then for any $x$ with $1 \leqq x \leqq s$ we may form the marked block

$$
b_{1}, \cdots, b_{x-1},\left[b_{x}\right], b_{x+1}, \cdots, b_{s} .
$$

But $B_{x}$ may also be interepreted for other values of $x$. For example, $B_{s+1}$ has such a meaning that for any $b$, the block $B_{s+1}, b$ is

$$
b_{1}, b_{2}, \cdots, b_{s},[b] \text {. }
$$

Extended Theorem. Suppose that $r_{0}, r_{1}, \cdots, r_{v-1}$, and $s$ are positive integers, and that $\alpha, \beta, \gamma$, and $\delta$ are real numbers greater than 1 . For $0 \leqq n \leqq v$, let $R_{n}$ stand for the sequence, with a marked term at or to the right of the middle,

$$
r_{v-1}, \cdots, r_{1}, r_{0}, r_{1}, \cdots,\left[r_{n}\right], \cdots, r_{v-1} .
$$

Then if $v-n$ is even, we shall have

$$
\left[\gamma, 1, s, R_{n}, s-1,1, \delta\right]<\left[\alpha, 1, s, R_{n}, s, 1, \beta\right],
$$

and provided $\alpha<4 s+3$ and $\beta<4 s+3$, also

$$
\left[\gamma, 1, s, R_{n}, s+1,1, \delta\right]>\left[\alpha, 1, s, R_{n}, s, 1, \beta\right] .
$$

If $v-n$ is odd, the inequalities are reversed.

Proof. For $n=0$, we have the preceding theorem. If the theorem is true for any $n$ with $0 \leqq n \leqq v-1$, then it is also true for $n+1$, by the lemma.

5. Lemmas on derivable sequences. Given a derivable sequence whose terms are ultimately $a$ and $a+1$. If

$$
b_{1}, b_{2}, \cdots, b_{n}
$$

is a block of the derived sequence, then by the corresponding block of the primitive sequence we shall mean

$$
a,(a+1)^{b_{1}}, a,(a+1)^{b_{2}}, a, \cdots, a,(a+1)^{b_{n}}, a .
$$

The block in the primitive corresponding to a block in the $m$ th derivative is found by repeated use of this rule. Notice that abutting blocks of a derivative correspond to overlapping blocks of the primitive. It is also clear that a symmetrical block in the $m$ th derivative cor- 
responds to a symmetrical block in the primitive.

Consider now a family of sequences, all having $m$ derivatives $(m \geqq 1)$, and all ultimately containing the same two numbers in the $p$ th derivative, for each $p$ with $0 \leqq p<m$. We wish to study the blocks in the primitives corresponding to certain types of blocks in the $m$ th derivatives. It is understood that the primitive sequences occurring in the following two lemmas belong to a family of the type mentioned. The letters $A$ and $B$ will stand for blocks of terms.

Lemma 1. Suppose that the block $b, B, b$ in an mth derivative corresponds to $a$ block $a, A, a$ in the primitive. Then if $c>b$ and $d>b$, blocks

$$
b, B, d ; \quad c, B, d ; \quad c, B, b
$$

in mth derivatives will correspond respectively to blocks in the primitives including

$$
a, A, a+1 ; \quad a+1, A, a+1 ; \quad a+1, A, a .
$$

Proof. Suppose first that $m=1$. Then to the block $b, B, b$ in the derivative corresponds the block

$$
a,(a+1)^{b}, \cdots,(a+1)^{b}, a
$$

in the primitive. To the block $b, B, d$ in the derivative corresponds the block

$$
a,(a+1)^{b}, \cdots,(a+1)^{d}, a
$$

in the primitive, which includes the block

$$
a,(a+1)^{b}, \cdots,(a+1)^{b}, a+1,
$$

as required. The other two cases are similar. If $m>1$, the proof follows easily by induction, using the result for $m=1$.

LEMMA 2. If a term $k$ in an mth derivative corresponds to a block $a, A, a$ in the primitive, then corresponding to any term greater than $k$ in an mth derivative will be a block in the primitive including $a, A, a+1$.

Proof. If $m=1$, then corresponding to a term $k$ in the derivative is a block

$$
a,(a+1)^{k}, a
$$

in the primitive, and corresponding to a term greater than $k$ is a block including

$$
a,(a+1)^{k}, a+1
$$


If $m>1$, the result follows by induction, using Lemma 1 .

6. Terminology. It will be sufficient to prove the main theorem for numbers $\xi$ such that $M^{+}(\xi) \geqq M^{-}(\xi)$. From the results of I, $\S 5$, it is clear that if such a number is critical, then it has the form

$$
\xi=\left[q_{0}, q_{1}, \cdots, q_{2 u}, 1, r_{1}, 1, r_{2}, 1, r_{3}, 1, \cdots\right],
$$

where the sequence $r_{1}, r_{2}, r_{3}, \cdots$ is either derivable or tends to a limit, finite or infinite. It follows furthermore that a number $\xi$ of the above form is critical if the sequence of $r$ 's tends to a limit. Thus to complete the proof of the main theorem, we need consider only numbers $\xi$ of the above form, with a derivable sequence of $r$ 's, that is, a sequence which ultimately contains just two numbers, $k$ and $k+1$. Furthermore, a number of this type is critical unless there is a number $\xi^{\prime}$ also of this type (and indeed with the same $k$ ), such that $M^{+}\left(\xi^{\prime}\right)<M^{+}(\xi)$ and $M^{-}\left(\xi^{\prime}\right)<M^{-}(\xi)$.

Corresponding to the number $\xi$, we have a sequence of $\lambda$ 's (see $\S 2$ ). Any $\lambda$ which is centered at a term $r_{n}$ will be called a major $\lambda$, whereas a $\lambda$ which is centered at a unit adjacent to a term $r_{n}$ will be called a minor $\lambda$. Notice that $M^{+}(\xi)$ is the limit superior of the major $\lambda$ 's, and $M^{-}(\xi)$ is the limit superior of the minor $\lambda$ 's. If the sequence of $r$ 's has an $m$ th derivative, then any major $\lambda$ centered at a term $r_{n}$ included in the block corresponding to a given term of the $m$ th derivative will be considered as belonging to this term, and likewise for a minor $\lambda$ centered at a unit adjacent to such a term $r_{n}$. Notice that the same $\lambda$ may belong to more than one term of the $m$ th derivative.

We shall now classify numbers $\xi$ of the above form according to properties of the sequence $r_{1}, r_{2}, r_{3}, \ldots$. If this sequence has $m$ derivatives $(m \geqq 1)$, and for $0 \leqq p<m$ the $p$ th derivative contains the numbers $a_{p}$ and $a_{p}+1$, then $\xi$ will be said to be of class

$$
\left(a_{0}, a_{1}, \cdots, a_{m-1}\right),
$$

or of the class $S$, if $S$ stands for this $m$-term sequence. If in addition the $m$ th derivative is $t_{1}, t_{2}, t_{3}, \cdots$, where

$$
\lim \inf t_{n}=k, \quad \lim \sup t_{n}=l,
$$

then $\xi$ will be said to be of class

$$
\left(a_{0}, a_{1}, \cdots, a_{m-1} ; k, l\right),
$$

or of class $(S ; k, l)$. Here $k$ and $l$ are integers or $\infty$, and may have any values in the intervals $0 \leqq k \leqq \infty, 1 \leqq l \leqq \infty$. Notice that if $0<p<m$, a number of class $S$ is certainly also of class 


$$
\left(a_{0}, a_{1}, \cdots, a_{p-1} ; a_{p}, a_{p}+1\right) .
$$

7. Fundamental inequality for $M^{+}(\xi)$. Considering numbers of a fixed class $S$, where $S$ is an $m$-term sequence, we ask how the major $\lambda$ 's depend on the terms of the $m$ th derived sequence.

THEOREM. Let $S$ be an $m$-term sequence $(m \geqq 1)$, and consider numbers $\xi$ of class $S$. Then every major $\lambda$ belonging to a term $l$ in an mth derived sequence is smaller than $L_{1}(S ; l)$, whereas to any term larger than $l$ in an mth derived sequence a major $\lambda$ greater than $L_{2}(S ; l)$ can be found, where $L_{1}(S ; l)$ and $L_{2}(S ; l)$ depend only on $S$ and $l$, and

$$
L_{2}(S ; l)>L_{1}(S ; l) \text {. }
$$

Proof. Corresponding to a term $l$ in an $m$ th derived sequence is a block $a, B, a$ in the primitive, where $B$ is symmetrical. Also, the block $B$ has the form

$$
b_{1}, b_{2}, \cdots, b_{s-1}, b_{s}
$$

where each $b_{x}$ is either $a$ or $a+1$. For $0 \leqq x \leqq s+1$, let $B_{x}$ stand for the marked sequence

$$
1, b_{1}, 1, b_{2}, 1, \cdots, 1,\left[b_{x}\right], 1, \cdots, 1, b_{s}, 1 .
$$

If $x=0$ or $x=s+1$, this is interpreted as in $\S 4$. It is clear that we may take

$$
L_{1}(S ; l)=\left[a+2,1, a, B_{x}, a, 1, a+2\right],
$$

for a suitable value of $x$ in the interval $0 \leqq x \leqq s+1$. Since the block $B$ is symmetrical, we may suppose $x \geqq(s+1) / 2$.

On the hand, to a term larger than $l$ in an $m$ th derived sequence corresponds a block in the primitive including $a, B, a+1(\$ 5$, Lemma 2). Thus we may take

$$
L_{2}(S ; l)=\left[a, 1, a, B_{x}, a+1,1, a\right],
$$

for any $x$ which we like; we shall use the same of $x$ as in defining $L_{1}(S ; l)$. By the extended theorem of $\S 4$, we see that

$$
L_{2}(S ; l)>L_{1}(S ; l) .
$$

Corollary. If $\xi$ is of class $(S ; k, l)$ and $\xi^{\prime}$ is of class $\left(S ; k^{\prime}, l^{\prime}\right)$ with $l^{\prime}>l$, then $M^{+}\left(\xi^{\prime}\right)>M^{+}(\xi)$.

8. Fundamental inequality for $M^{-}(\xi)$. Considering numbers of a fixed class $S$, where $S$ is an $m$-term sequence, we ask how the minor $\lambda$ 's depend on the terms of the $m$ th derived sequence. 
THEOREM 1. Let $S$ be an $m$-term sequence ( $m \geqq 1$ ), and consider numbers $\xi$ of class $S$. Then every minor $\lambda$ belonging to two consecutive terms, both greater than $k$, in an mth derived sequence is smaller than $K_{1}(S ; k)$, whereas to any pair of consecutive terms in an mth derived sequence, of which the smaller is equal to $k, a$ minor $\lambda$ belonging to both and greater than $K_{2}(S ; k)$ can be found, where $K_{1}(S ; k)$ and $K_{2}(S ; k)$ depend only on $S$ and $k$, and

$$
K_{2}(S ; k)>K_{1}(S ; k) .
$$

Proof. Corresponding to a hypothetical block $k, k$ in an $m$ th derivative, is a block $a, C, a$ in the primitive, where $C$ is symmetrical. The block $C$ has the form

$$
c_{1}, c_{2}, \cdots, c_{t-1}, c_{t},
$$

each $c_{x}$ being either $a$ or $a+1$. We may choose $w$ so that just those terms $c_{x}$ with $w+1 \leqq x \leqq t-w$ correspond to both of the $k$ 's. For $0 \leqq x \leqq t$, let $C_{x}$ stand for the marked sequence

$$
1, c_{1}, 1, c_{2}, 1, \cdots, 1, c_{x},[1], c_{x+1}, 1, \cdots, 1, c_{t}, 1 .
$$

Now by $\S 5$, Lemma 1 , to a block $k_{1}, k_{2}$ in an $m$ th derivative, where $k_{1}>k$ and $k_{2}>k$, will correspond a block in the primitive including $a+1, C, a+1$. The terms of the primitive corresponding to both $k_{1}$ and $k_{2}$ are exactly $c_{w+1}, \cdots, c_{t-w}$. Hence we may take

$$
K_{1}(S ; k)=\left[a, 1, a+1, C_{x}, a+1,1, a\right],
$$

for some value of $x$ with $w \leqq x \leqq t-w$. Because of the symmetry of $C$, we may suppose $x \geqq t / 2$.

Now suppose that a pair of consecutive terms, $k_{0}$ and $k$, are given in an $m$ th derivative, with $k_{0} \geqq k$. We may suppose that $k_{0}$ precedes $k$. Then to the block $k_{0}, k$ in the $m$ th derivative there will correspond a block in the primitive including either $a+1, C, a$ or $a, C, a$ ( $\$ 5$, Lemma 1). The terms corresponding to both $k_{0}$ and $k$ will again be $c_{w+1}, \cdots, c_{t-w}$. Hence we may take

$$
K_{2}(S ; k)=\left[a+2,1, a+1, C_{x}, a, 1, a+2\right],
$$

with the same $x$ used before. The inequality

$$
K_{2}(S ; k)>K_{1}(S ; k)
$$

follows from the extended theorem of $\S 4$.

THEOREM 2. Let $S$ be an $m$-term sequence $(m \geqq 1)$, and consider numbers $\xi$ of class $S$. Then every minor $\lambda$ belonging to a single term (but 
not the first term) of the mth derivative is smaller than $K_{1}^{*}(S)$, whereas to any pair of consecutive terms a corresponding minor $\lambda$ can be found larger than $K_{2}^{*}(S)$, where $K_{1}^{*}(S)$ and $K_{2}^{*}(S)$ depend only on $S$, and

$$
K_{2}^{*}(S)>K_{1}^{*}(S) \text {. }
$$

Proof. Consider first the case $m=1$, and let $S=(a)$. Notice that a minor $\lambda$ belonging to some term of the first derivative beyond the first term will belong to two consecutive terms of the first derivative if and only if it is centered at a unit adjacent to an $a$. Hence we may take

$$
K_{1}^{*}(a)=[a, 1, a+1,[1], a+1,1, a]
$$

and

$$
K_{2}^{*}(a)=[a+2,1, a+1,[1], a, 1, a+2] .
$$

The inequality $K_{2}^{*}(a)>K_{1}^{*}(a)$ follows from the theorem of $\$ 4$.

Now assume the theorem for numbers of class $S$, and prove for numbers of class $(S, k)$, where $(S, k)$ denotes the $(m+1)$-term sequence obtained by adjoining $k$ to $S$. We shall show that we may take

$$
K_{1}^{*}(S, k)=K_{1}(S ; k), \quad K_{2}^{*}(S, k)=K_{2}(S ; k),
$$

where $K_{1}(S ; k)$ and $K_{2}(S ; k)$ are quantities satisfying Theorem 1 . Indeed, any $\lambda$ belonging to a single term (not the first) of the $(m+1)$ st derivative can belong only to terms $k+1$ of the $m$ th derivative. By the inductive hypothesis, the largest such minor $\lambda$ 's belong to two consecutive such terms; and by Theorem 1, such $\lambda$ 's satisfy

$$
\lambda<K_{1}(S ; k) \text {. }
$$

On the other hand, any pair of consecutive terms of the $(m+1)$ th derivative have in common all $\lambda$ 's belonging to a certain term $k$ of the $m$ th derivative, and hence by Theorem 1 there is certainly some minor $\lambda$ belonging to both which satisfies

$$
\lambda>K_{2}(S ; k) \text {. }
$$

From Theorems 1 and 2, the following result is clear.

Corollary. If $\xi$ is of class $(S ; k, l)$ and $\xi^{\prime}$ of class $\left(S ; k^{\prime}, l^{\prime}\right)$ with $k^{\prime}<k$, then $M^{-}\left(\xi^{\prime}\right)>M^{-}(\xi)$.

9. Proof of the main theorem. Two more lemmas will be needed for the proof of the main theorem.

LEMma 1. Let $S$ be an m-term sequence ( $m \geqq 1)$. If $\xi_{1}$ is of class 
$\left(S ; k_{1}, l_{1}\right)$ and $\xi_{2}$ of class $\left(S ; k_{2}, l_{2}\right)$, where $k_{1} \leqq l_{1} \leqq k_{2} \leqq l_{2}$, then

$$
M^{+}\left(\xi_{1}\right) \leqq M^{+}\left(\xi_{2}\right), \quad M^{-}\left(\xi_{1}\right) \geqq M^{-}\left(\xi_{2}\right) .
$$

Proof. If $k_{1}=l_{1}=k_{2}=l_{2}$, then evidently

$$
M^{+}\left(\xi_{1}\right)=M^{+}\left(\xi_{2}\right), \quad M^{-}\left(\xi_{1}\right)=M^{\sim}\left(\xi_{2}\right) .
$$

Hence we may suppose that $k_{1}<l_{2}$. It will be sufficient to show that if we are given terms $j_{1}$ and $j_{2}$, sufficiently far out in the $m$ th derived sequences for $\xi_{1}$ and $\xi_{2}$, respectively, then the largest major $\lambda$ belonging to $j_{2}$ is greater than any major $\lambda$ belonging to $j_{1}$, whereas the largest minor $\lambda$ belonging to $j_{1}$ is greater than any minor $\lambda$ belonging to $j_{2}$. If $j_{1}<j_{2}$, this follows for the major $\lambda$ 's from the theorem of $\S 7$, and for minor $\lambda$ 's from $\S 8$, Theorems 1 and 2 .

Suppose now that $j_{1}=j_{2}$. Match up the two $m$ th derived sequences so that these terms are paired, and continue the pairing to the left and right until unequal terms are encountered. This will give us blocks $g_{1}, J, h_{1}$ and $g_{2}, J, h_{2}$ in the two $m$ th derivatives, with $j_{1}$ and $j_{2}$ appearing at the same position in the block $J$, and with $g_{1}<g_{2}, h_{1}<h_{2}$. To the block $g_{1}, J, h_{1}$ in the $m$ th derivative will correspond a block $a, D, a$ in the primitive. By $\$ 5$, Lemma 1 , the block in the primitive corresponding to $g_{2}, J, h_{2}$ will include $a+1, D, a+1$. It is then clear that of a pair of major $\lambda$ 's, one for $\xi_{1}$ and the other for $\xi_{2}$, centered at the same position relative to the block $D$, the second will be the larger. For a similar pair of minor $\lambda$ 's, the first will be the larger. Thus, in particular, the desired relation is established between the $\lambda$ 's belonging to $j_{1}$ and $j_{2}$.

LEMMA 2. Let $S$ be an $m$-term sequence ( $m \geqq 1)$. If $\xi$ is of class $S$, and

$$
M^{+}\left(\xi^{\prime}\right)<M^{+}(\xi), \quad M^{-}\left(\xi^{\prime}\right)<M^{-}(\xi),
$$

then $\xi^{\prime}$ is also of class $S$.

Proof. For $S=(a)$, the result follows from I, $\S 5$. Now assume the theorem for numbers of class $S$, and prove for numbers of class $(S, k)$, where $(S, k)$ denotes the $(m+1)$-term sequence obtained by adjoining $k$ to $S$. We are given a number $\xi$ of class $(S, k)=(S ; k, k+1)$. The inductive hypothesis shows that $\xi^{\prime}$ is of class $S$. The corollaries of $\S \S 7-8$ show that $\xi^{\prime}$ must be of class $(S ; k, k),(S ; k+1, k+1)$, or $(S ; k, k+1)$. The first two possibilities are eliminated by Lemma 1 . Hence $\xi^{\prime}$ must be of class $(S ; k, k+1)=(S, k)$.

Proof of MAIN THEOREM. Suppose first that $\xi$ has exactly $m$ derived sequences $(m \geqq 1)$, and is of class $S$, where $S$ is an $m$-term sequence. To test whether $\xi$ is critical, according to Lemma 2 we need only find 
whether there is a number $\xi^{\prime}$ of class $S$ such that

$$
M^{+}\left(\xi^{\prime}\right)<M^{+}(\xi), \quad M^{-}\left(\xi^{\prime}\right)<M^{-}(\xi)
$$

Now $\xi$ must be of class $(S ; k, l)$ for some $k$ and $l$, and indeed $l=k$ or $l>k+1$, since $\xi$ has exactly $m$ derived sequences. If $\xi^{\prime}$ is of class $\left(S ; k^{\prime}, l^{\prime}\right)$, then by the corollaries of $\S \S 7-8$, we must have $k \leqq k^{\prime} \leqq l^{\prime} \leqq l$.

If $l=k \leqq \infty$, we must have $k=k^{\prime}=l^{\prime}=l$, whence

$$
M^{+}\left(\xi^{\prime}\right)=M^{+}(\xi), \quad M^{-}\left(\xi^{\prime}\right)=M^{-}(\xi) .
$$

In other words, no suitable $\xi^{\prime}$ can be chosen, hence $\xi$ is critical. If $l>k+1$, any $\xi^{\prime}$ of class $(S ; j, j)$ with $k<j<l$ serves as a comparison number to show that $\xi$ is not critical. Thus when $\xi$ has a last derived sequence, it is critical only when this sequence has a limit, finite or infinite.

Suppose on the other hand that $\xi$ has infinitely many derived sequences. Let the numbers appearing in the $p$ th derivative be $a_{p}$ and $a_{p}+1$. Let $\xi_{m}$ be any number of class

$$
\left(a_{0}, a_{1}, \cdots, a_{m-1} ; 1,1\right) .
$$

Then $\xi_{m}$ is critical. It is easily seen that

$$
M^{+}\left(\xi_{m}\right) \rightarrow M^{+}(\xi), \quad M^{-}\left(\xi_{m}\right) \rightarrow M^{-}(\xi) .
$$

Hence $\xi$ is also critical.

University of California 